\title{
Explanation of Pressure Effect for High Temperature Superconductors Using Pressure Dependent Schrodinger Equation and String Theory
}

\author{
Einas Mohamed Ahmed Mohamed ${ }^{1,2}$, Nagwa Idriss Ali Ahmed ${ }^{2,3}$, Musa Ibrahim Babiker Hussein ${ }^{4,5}$, \\ Rasha Abd Elhai Mohammad Taha ${ }^{6,7}$, Mohammed Idriss Ahmed ${ }^{7}$, Mubarak Dirar Abd-Alla ${ }^{7}$ \\ ${ }^{1}$ Department of Physics, University College (Turaba), Taif University, Taif, KSA; ${ }^{2}$ Department of Physics and \\ Mathematics, Hantoub Faculty of Education, Algezira University, Wad Madaniin-Hantoub, Sudan; ${ }^{3}$ Department of \\ Physics, Faculty of Science \& Art (Dariyah), Qassim University, Al-Mulida, KSA; ${ }^{4}$ Department of Physics and \\ Mathematics, Faculty of Education, Albutana University, Rufaa, Sudan; ${ }^{5}$ Department of Physics, Faculty of Science \\ \& Art, Dariyah University, Buljurashi, KSA; ${ }^{6}$ Department of Physics, College of Science, Majmaah University, \\ Majmaah, KSA; ${ }^{7}$ Department of Physics, Faculty of Science, Sudan University of Science and Technology, \\ Khartoum, Sudan
}

Correspondence to: Mohammed Idriss Ahmed, garaof@yahoo.com

Keywords: Pressure Dependent Schrodinger Equation, Superconductivity, Critical Temperature Pressure, High Temperature Superconductor

Received: October 10, $2019 \quad$ Accepted: January 17, $2020 \quad$ Published: January 20, 2020

Copyright $\odot 2020$ by author(s) and Scientific Research Publishing Inc.

This work is licensed under the Creative Commons Attribution International License (CC BY 4.0).

http://creativecommons.org/licenses/by/4.0/

\section{(c) (i) Open Access}

\section{ABSTRACT}

A pressure dependent Schrodinger equation is used to find the conditions that lead to superconductivity. When no pressure is exerted, the superconductor resistance vanishes beyond a critical temperature related to the repulsive force potential of the electron gass, where one assuming the electron total energy to be thermal, where applying mechanical pressure destroys Sc when it exceeds a certain critical value. However when the electron total energy is an assumed to be that of the free electron model and that the pressure is thermal and mechanical, the situation is different. The quantum expression for resistance shows that the increase of mechanical pressure increases the critical temperature. Such phenomenon is observed in high temperature cupper group.

\section{INTRODUCTION}

Superconductivity $(\mathrm{Sc})$ phenomenon is one of the most interesting properties of bulk matter. In this phenomenon, the resistance to electric current vanishes beyond a certain critical temperature [1].

This leads to generation of powerful magnetic field that used in a wide variety of applications.

For instance, the magnetic property is used in magnetic resonance imaginary (MRI), super magnetic 
trains and in generating powerful electric energy [2].

The wide spread of Sc applications face a disaster problem. This problem is related to the fact that the operating temperature of Sc materials are far beyond $100 \mathrm{k}$, which is much lower than the ambient tem-

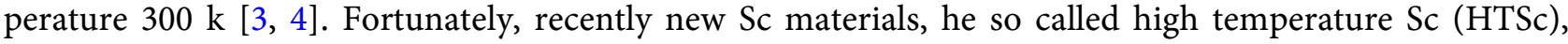
were discovered.

They can operate at temperatures above $130 \mathrm{k}$ as $[5,6]$. To reach the ambient temperature operation, one needs a well-defined model that gives a clear path way that shows how to select compounds to increase the critical temperature to be above the ambient temperature.

Unfortunately there are many problems associated with (HTSc). They suffer from some long standing problem like pressure problem and isotope problem. In the former one, the application of pressure on some compound can increase or decrease the critical temperature.

In the latter one, the replacement of some compound constituent by their isotope changes the critical temperature $[7,8]$.

Fortunately, some models were proposed to solve some of these problem. Some models solve pressure problem, while other models try to explain magnetic destruction of Sc $[9,10]$.

This needs new alternatives that leads to a well define model that can solve all Sc problems. This paper is concerned with construction and development of a new that can be promoted to solve the problems associated with the Sc. Section 2 is concerned with constructing a new model based on string theory to explain the pressure effect. The discussion and conclusion are exhibited in Sections 3 and 4.

\section{PREVIOUS WORK}

In a work done by many authors [11], an energy dependent expression on the potential $V$ and pressure $P$ was obtained from the plasma equation:

$$
\begin{gathered}
v m \frac{\mathrm{d} v}{\mathrm{~d} x}=m \frac{\mathrm{d} x}{\mathrm{~d} t}=-\nabla P-\nabla V=-\mathrm{d}\left(\frac{P+V}{\mathrm{~d} x}\right) \\
\frac{\mathrm{d}}{\mathrm{d} x}\left(\frac{1}{2} m v^{2}+V+P\right)=0
\end{gathered}
$$

Thus:

$$
K E+V+P=\text { Constant }
$$

This constant of motion, which is shown to be standing for the energy of the system, is given by:

$$
E=K E+V+P
$$

\section{THE PRESSURE DEPENDENT SCHRODINGER MODEL}

The ordinary Schrodinger equation is given, according to (3), by:

$$
i \hbar \frac{\mathrm{d} \Psi}{\mathrm{d} t}=-\frac{\hbar^{2}}{2 m} \nabla^{2} \Psi+V \Psi+P \Psi
$$

The time independent equation can be found by suggesting the wave function (wf) to be:

$$
\Psi=u \mathrm{e}^{-i \omega_{0} t}=u(r) \mathrm{e}^{-i \omega_{0} t}
$$

A direct substitution of (5) in (4) yields:

$$
E_{0} u=\hbar \omega_{0} u=-\frac{\hbar^{2}}{2 m} \nabla^{2} u+V u+P \Psi
$$

Consider an electron acting a harmonic oscillator subjected to a constant potential $V_{1}$. For electron 
Schrodinger Equation (6) is written as:

$$
E_{0} u=-\frac{\hbar^{2}}{2 m} \nabla^{2} u+\frac{1}{2} k x^{2} u+V u+P \Psi
$$

Try solution of the form:

$$
\begin{gathered}
u=A \mathrm{e}^{-\alpha x^{2}} \\
\nabla u=-2 \alpha x u \\
\nabla^{2}=-2 \alpha u-2 \alpha x \nabla u \\
\nabla^{2} u=-2 \alpha u+4 \alpha^{2} x^{2} u
\end{gathered}
$$

In view of Equation (3) c consider the potential $V$ to result from repulsive crystal potential of the electron gass $V_{0}$ beside the mechanical pressure $P_{0}$.

In this case:

$$
\begin{gathered}
V=\frac{1}{2} k x^{2}+V_{0} \\
P=-P_{0}
\end{gathered}
$$

Here the pressure is exerted on the system by surrounding media, inserting (8) in (7) gives:

$$
E_{0} u=-\frac{\hbar^{2}}{2 m}\left(-2 \alpha+4 \alpha^{2} x^{2}\right) u+\left(\frac{1}{2} k x^{2}+V_{0}-V_{p}\right) u
$$

Equating the coefficients of $u$ and $x^{2} u$ yields:

$$
E_{0}=\frac{\hbar^{2} \alpha}{2 m}+V_{0}-V_{p}=\frac{\hbar^{2} \alpha}{m}+V_{0}-P_{0}
$$

And:

$$
\frac{2 \hbar^{2} \alpha^{2}}{m}=\frac{1}{2} k=\frac{1}{2} m \omega^{2}
$$

From (12), one gets:

$$
\alpha^{2}=\frac{m^{2} \omega^{2}}{4 \hbar^{2}}=\frac{m^{2} c^{4}}{4 \hbar^{4} c^{4}}
$$

where one assumes that the energy satisfies Einstein and Max plank relations, i.e.:

$$
E=m c^{2}=\hbar \omega
$$

Thus:

$$
\begin{gathered}
\alpha^{2}=\frac{(\hbar \omega)^{4}}{4 \hbar^{4} c^{4}}=\frac{1}{4}\left(\frac{\omega}{c}\right)^{4}=\frac{1}{4}\left(\frac{c k}{c}\right)^{4}=\frac{1}{4} k^{4} \\
\alpha=\frac{1}{2} k^{2}
\end{gathered}
$$

There for Equation (11) gives:

$$
E_{0}=\frac{\hbar^{2} k^{2}}{2 m}+V_{0}-V_{P}=\frac{\hbar^{2} K^{2}}{2 m}+V_{0}-P_{0}
$$

Thus the momentum is given by:

$$
m v=\hbar k=\sqrt{2 m\left(E_{0}-V_{0}+V_{p}\right)}
$$


This relation can be used to define a quantum resistance by using the relation:

$$
R=\frac{U}{I}=\frac{V}{e I}=\frac{V}{\text { enevA }}=\frac{m v^{2}}{2 n e^{2} v A}=\frac{m v}{2 n e^{2} A}
$$

With $U, I, V$ and $n$ standing for the electric potential, current potential energy and concentration respectively:

$$
R=R_{r}+i R_{i}=R_{s}+i R_{i}
$$

Here $R_{r}, R_{s}, R_{i}$ stands for real, superconductor and imaginary resistance respectively.

When no pressure was applied:

$$
P_{0}=V_{0}=0
$$

In view of Equations (17) and (18), one gets

$$
R=\frac{\hbar k}{2 n e^{2} A}
$$

Using Equations (17), (20) in (21) yields:

$$
R=\frac{\sqrt{2 m\left(E_{0}-V_{0}\right)}}{2 n e^{2} A}
$$

Assume a gain that the energy $E_{0}$ is in the form of thermal energy:

$$
E_{0}=K T
$$

Using (23) in (22) gives:

$$
R=\frac{\sqrt{2 m\left(K T-V_{0}\right)}}{2 n e^{2} A}
$$

The resistence $R$ become pure imaginary when:

$$
K T<V_{0}
$$

Define the critical temperature to be satisfying the relation:

$$
V_{0}=K T_{c}
$$

Thus Equation (25) requires:

$$
K T<K T_{c}
$$

Hence:

$$
T<T_{c}
$$

Thus according to Equations (19) and (24) one gets:

$$
R=R_{i}, R_{s}=0
$$

However when the pressure was applied. Such that: $P_{0}>V_{0}$

$$
P_{0} \geq K T_{c}
$$

Using also Equations (17), (16), (21), (23), (26) one gets:

$$
R=\frac{\sqrt{2 m\left(K T-K T_{c}+P_{0}\right)}}{2 n e^{2} A}
$$

In this case in view of Equations (29) and (30), one gets: 


$$
R_{i}=0
$$

For all values of $T$, i.e. for:

$$
T \geq 0
$$

Therefore according to Equations (19) \& (31) the superconducting resistance is no longer equal to zero, i.e.:

$$
R=R_{s} \neq 0
$$

For all values of $T$ greater than zero.

Thu applying pressure such that it exceeds a certain critical value gives by (29) and (26) destroys Sc. The new critical decreases upon increasing pressure, when:

$$
T_{C}=T_{C}-P_{0}
$$

Another approach can be suggested by assuming that the pressure result from a thermal one $K T$ exerted by the surrounding. In this case Equation (3) takes the form:

$$
E=K E+V+K T-P_{0}
$$

Assuming also the existence of very large attractive force on electrons due to the ionic cores:

$$
V=-V_{0}
$$

Thus one can write:

$$
K E+V=K E-V_{0}=-V_{1}
$$

where the potential energy is assumed to be much large than the kinetic energy. Using nearly electron model for solids:

$$
E=\frac{\hbar^{2} k^{2}}{2 m^{*}}
$$

Thus Equations (35), (36), (37) and (38) gives:

$$
\hbar k=\sqrt{2 m^{*}\left(K T-V_{1}-P_{0}\right)}
$$

Define now the critical temperature to satisfy:

$$
K T_{c}=V_{1}+P_{0}
$$

Thus:

$$
\hbar k=\sqrt{2 m^{*} k\left(T-T_{c}\right)}
$$

According to Equations (17), (18), (19) and (41):

$$
R=R_{i}+i R_{s}=\frac{\sqrt{2 m^{*} k\left(T-T_{c}\right)}}{2 n e^{2} A}
$$

Thus for all:

$$
\begin{aligned}
& T \leq T_{c} \\
& R=R_{I} \\
& R_{s}=0
\end{aligned}
$$

It is very interesting to note that Equation (40), shows that the critical temperature can be increased by increasing the applied mechanical pressure as observed for $\mathrm{Cu}$. 


\section{DISCUSSION}

Pressure dependent Schrodinger equation [see Equation (14)] is used to find a useful expression for energy, wave number and momentum.

These expressions were obtained by treating electrons as vibrating strings subjected to repulsive electron force and mechanical pressure.

Using the ordinary expression for resistance a useful expression of quantum resistance is obtained [see Equations (18), (21)] through the momentum and wave number. Assuming first electron energy to be purely thermal [Equations (23)], the Sc resistance [Equations (24)-(28)] vanishes beyond a critical temperature in the absence of the external pressure. This a grees with experiment. Applying external pressure destroys Sc when the pressure exceeds a certain critical value [see Equations (29)-(34)]. The pressure here decreases the critical temperature.

When one assume the pressure to be resulting from electron gas thermal pressure beside external mechanical pressure [see Equation (35)], the critical temperature in (40) increases upon increasing pressure. This can thus explains the behavior of high temperature Sc copper group.

\section{CONCLUSION}

The pressure dependent Schrodinger equation can be used to obtain a useful expression of quantum resistance. This expression can be used to explain the effect of external mechanical pressure on high temperature Sc. It can explain why pressure decreases or increases sometimes the critical temperature.

\section{CONFLICTS OF INTEREST}

The authors declare no conflicts of interest regarding the publication of this paper.

\section{REFERENCES}

1. Annett, J.F. (2006) Superconductivity, Superfluid's and Condense States, OVP, Campridge.

2. Poole Jr., C.P., Afrach, H. and Creswick, R.J. (2007) Superconductivity. Academic Press, London.

3. Jay Kumarsaxena, A. (2009) High Temperature Superconductors, Rewa, India.

4. Hammed, I.A.I., Dirar, M., Atta, N.O., Taha, R.A., Haroun, Kh.M. and Elgani, R.A. (2017) Pressure Effect on Superconducting Critical Temperature According to String Model. International Journal of Fluid Mechanics \& Thermal Sciences, 3, 70-74. https://doi.org/10.11648/j.ijfmts.20170306.12

5. Abdalrahman, R.I., Taha, R.A.E.M., Attia, I.A. and Allah, M.D.A. (2016) New Derivation of Simple Josephson Effect Relation Using New Quantum Mecjanical Equation. Natural Science, 8, 85-88.

https://doi.org/10.4236/ns.2016.83011

6. Mubark Dirar, A., As, A., Ahmed, Z., Asma, M.E., Rawia, A. and Amel, A.A. (2006) Effect of Magnetic Field on Superconducting Complex Resistance According to Quantum Mechanics. Global Journal of Engineering Science and Researches, 3.

7. Dirar, M., Einas, M. and Elhouri, S.A. (2015) Relation between Critical Temperature and Superconductivity Zero Resistance According to Quantum Laws. International Journal of Management Science and Engineering Management, 5.

8. Zakaria, A., Asma, A., Dirar, M., Asma, M.E. and Amel, A. (2016) Quantum Effect of Magnetic Field in Destroying Superconductivity. International Journal of Management Science and Engineering Management, 2.

9. Scott, A.C. (2003) Nonlinear Science: Emergence \& Dynamics of Coherent Structures. 2nd Edition, Oxford University Press, New York. 
10. Sulem, C. and Sulem, P.-L. (1999) The Nonlinear Schrodinger Equation: Self-Focusing and Wave Collapse. Springer-Verlag, New York.

11. Karpman, V.I. (1975) Nonlinear Waves in Dispersive Media. Pergamon, Oxford. https://doi.org/10.1016/B978-0-08-017720-5.50005-1 